\title{
Relationship of macrophage migration inhibitory factor levels in PBMCs, lesional skin and serum with disease severity and activity in vitiligo vulgaris
}

\author{
Lei $\mathrm{Ma}^{1 *}$, Hai-Bo Xue ${ }^{2 *}$, Xiu-Hao Guan ${ }^{3 *}$, Chun-Mei Shu ${ }^{1}$, Yu-Jie Zhang ${ }^{1}$ \\ Jun-Hua Zhang ${ }^{1}$ and Rong-Zhen $\mathrm{An}^{1}$ \\ ${ }^{1}$ Department of Dermatology, Binzhou Medical University Hospital, Binzhou, China \\ ${ }^{2}$ Department of Endocrinology and Metabolism, Binzhou Medical University Hospital, Binzhou, China \\ ${ }^{3}$ State Key Department of Dermatology, No. 1 Hospital of China Medical University, Shenyang, China
}

\begin{abstract}
Melanocyte loss in vitiligo vulgaris is believed to be an autoimmune process. Macrophage migration inhibitory factor (MIF) is involved in many autoimmune skin diseases. We determined the possible role of MIF in the pathogenesis of vitiligo vulgaris, and describe the relationship between MIF expressions and disease severity and activity. Serum MIF concentrations and mRNA levels in PBMCs were measured in 44 vitiligo vulgaris patients and 32 normal controls, using ELISA and real-time RTPCR. Skin biopsies from 15 patients and 6 controls were analyzed by real-time RT-PCR. Values are reported as median (25th75th percentile). Serum MIF concentrations were significantly increased in patients [35.81 (10.98-43.66) ng/mL] compared to controls [7.69 (6.01-9.03) ng/mL]. MIF mRNA levels were significantly higher in PBMCs from patients [7.17 (3.59-8.87)] than controls [1.67 (1.23-2.42)]. There was also a significant difference in MIF mRNA levels in PBMCs between progressive and stable patients [7.86 (5.85-9.13) vs 4.33 (2.23-8.39)] and in serum MIF concentrations [40.47 (27.71-46.79) vs 26.80 (10.5536.07) $\mathrm{ng} / \mathrm{mL}$ ]. In addition, the vitiligo area severity index scores of patients correlated positively with changes of both serum MIF concentrations $(r=0.488)$ and MIF mRNA levels in PBMCs $(r=0.426)$. MIF mRNA levels were significantly higher in lesional than in normal skin [2.43 (2.13-7.59) vs $1.18(0.94-1.83)]$ and in patients in the progressive stage than in the stable stage [7.52 (2.43-8.84) vs 2.13 (1.98-2.64)]. These correlations suggest that MIF participates in the pathogenesis of vitiligo vulgaris and may be useful as an index of disease severity and activity.
\end{abstract}

Key words: Migration inhibitory factor; Vitiligo vulgaris

\section{Introduction}

Vitiligo is a chronic skin disorder characterized by progressive loss of functional melanocytes, which results in depigmented macules in skin, hair, and mucous membranes. The precise etiology of vitiligo remains obscure, and the prevailing hypotheses regarding the pathomechanisms of the condition are autoimmune, genetic, neural, and/or biochemical factors (1-6). Among different types of vitiligo, melanocyte loss in vitiligo vulgaris is now widely believed to be an autoimmune process, and one of the strongest factors supporting an autoimmune origin of vitiligo vulgaris is its epidemiological association with other autoimmune diseases $(2,6)$.
Macrophage migration inhibitory factor (MIF) has been originally identified as a lymphokine, which can concentrate macrophages at inflammation loci. MIF is also one of the immunoregulatory cytokines involved in macrophage and T-cell activation and, conversely, macrophages and T cells are the primary source of MIF. MIF is known to be involved in immune-mediated diseases, and may also play a pivotal role in many autoimmune skin diseases, such as systemic lupus erythematosus, systemic sclerosis, atopic dermatitis, psoriasis vulgaris, bullous pemphigoid, etc. (7-10). However, little is known about the contribution of MIF to vitiligo vulgaris.

Correspondence: Hai-Bo Xue, Department of Endocrinology and Metabolism, Binzhou Medical University Hospital, 661 Second Huanghe Street, Binzhou, 256603, China. E-mail: xue_haibo@hotmail.com

${ }^{*}$ These authors contributed equally to this study.

Received February 13, 2012. Accepted September 6, 2012. First published online September 21, 2012. 
To elucidate the involvement of MIF in the pathogenesis of vitiligo vulgaris, we measured MIF mRNA levels in peripheral blood mononuclear cells (PBMCs) and skin biopsies, and serum MIF concentrations in vitiligo vulgaris patients and normal controls. We also analyzed the possible relationship between MIF expressions and the severity and activity of vitiligo vulgaris.

\section{Subjects and Methods}

\section{Subjects}

Forty-four vitiligo vulgaris patients (23 males and 21 females ranging in age from 18 to 56 years, with a mean age of 30.3 years) were enrolled in the study. Patients were excluded if they had diabetes mellitus, thyroiditis, pernicious anemia, psoriasis, atopic dermatitis, connective tissue disease, and other autoimmune/inflammatory diseases, and the patients should not have received systemic corticosteroids or other immunosuppressants within the last 6 months. Patients were divided into two groups on the basis of disease activity, namely 28 subjects in the progressive stage (active vitiligo, in which new areas of depigmentation, enlargement of the area of depigmentation or both were observed during the previous month) and 16 subjects in the stable stage (inactive vitiligo, in which no new depigmentation or enlargement of depigmentation had been observed for more than 3 months). The severity of vitiligo vulgaris was assessed with the vitiligo area severity index (VASI) (11).

The control group consisted of 32 healthy subjects ( 17 males and 15 females ranging in age from 22 to 50 years, with a mean age of 33.4 years). Subjects with systemic disease, current infection or immunosuppression, and pregnant or lactating women were excluded from the study. The age and gender ratio were well matched in the two groups.

The Human Ethics Committee of Binzhou Medical University approved the study, and written informed consent was obtained from all participants.

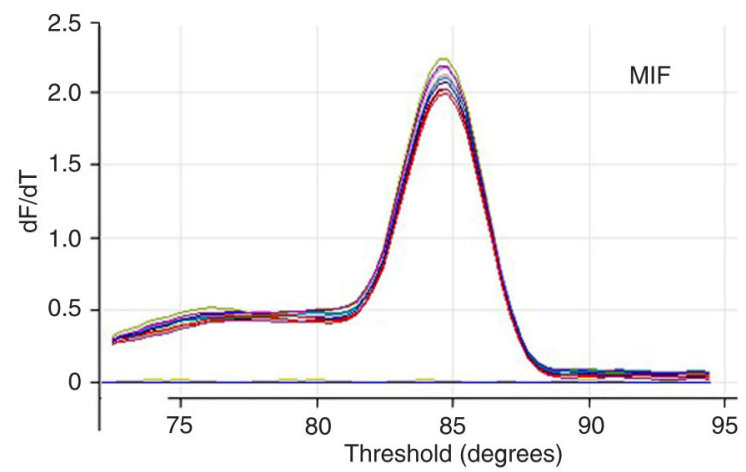

\section{Methods}

Blood was taken from all participants, after an overnight fast, in the morning between 8:00 and 9:00 am. Serum was centrifuged within 30 min of blood collection and stored at $-80^{\circ} \mathrm{C}$ for further serologic testing (ELISA kit, R\&D Systems, Inc., USA). PBMCs were collected by Ficoll-Hypaque density gradient centrifugation. Biopsies of lesional skin were obtained from 15 patients ( 8 in the progressive stage and 7 in the stable stage) and biopsies of normal skin were obtained from 6 control subjects. Total RNA was isolated from PBMCs and biopsy tissues with Trizol reagent (Invitrogen, USA) following manufacturer instructions. The quality of the RNA samples was assessed by inspecting the $28 \mathrm{~S}$ and $18 \mathrm{~S}$ bands after $1.5 \%$ agarose gel electrophoresis. The 260/280 absorbance ratio was between 1.9 and 2.0 for each RNA sample. A reverse transcriptase kit (PrimeScript ${ }^{\mathrm{TM}} \mathrm{RT}$ reagent kit, TaKaRa, Japan) was used for complementary DNA (cDNA) synthesis. Real-time RT-PCR was performed using a Rotor-Gene 3000 (Corbett Research, Australia) and mRNA levels were quantified using SYBR Premix Ex Taq $^{\mathrm{TM}}$ II (TaKaRa). Primers were designed by the Internet Primer Design Software and synthesized by Shenggong Company (Shanghai, China). MIF primers were: 5'-ACCAGCTCATGGCCTTCG-3' (forward) and 5'-CTTGCTGTAGGAGCGGTT-3' (reverse). $\beta$-actin was used as an endogenous reference with primers: 5'AGTTGCGTTACACCCTTTCTTG-3' (forward) and 5'TCACCTTCACCGTTCCAGTTT-3' (reverse). Data were analyzed using the Rotor-Gene Real-Time Analysis Software 6.0 and the melting curves of MIF and $\beta$-actin are shown in Figure 1.

\section{Statistical analysis}

Data were analyzed statistically by SPSS for Windows (version 17.0; SPSS Inc., USA). Values are reported as median (25th-75th percentile). The Mann-Whitney U-test and Spearman's correlation were used for statistical analysis, with the level of significance set at $\mathrm{P}<0.05$.

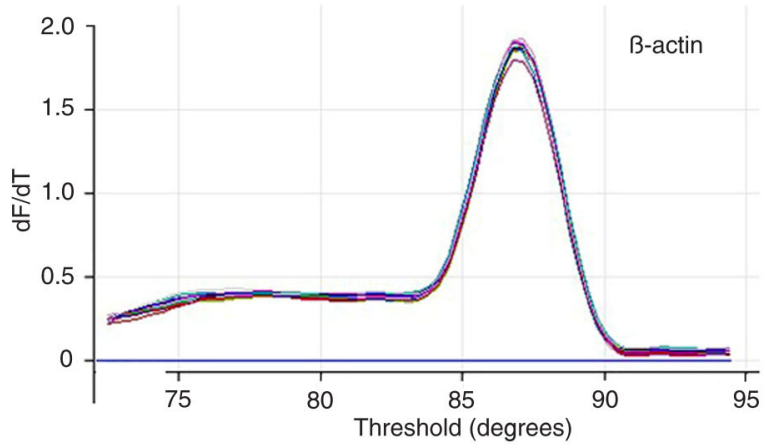

Figure 1. Melting curves of migration inhibitory factor (MIF) mRNA and $\beta$-actin mRNA. The melting temperature is uniform and the shapes of the peaks are sharp, which indicate that MIF and $\beta$-actin are the only amplicons in the amplification products. 


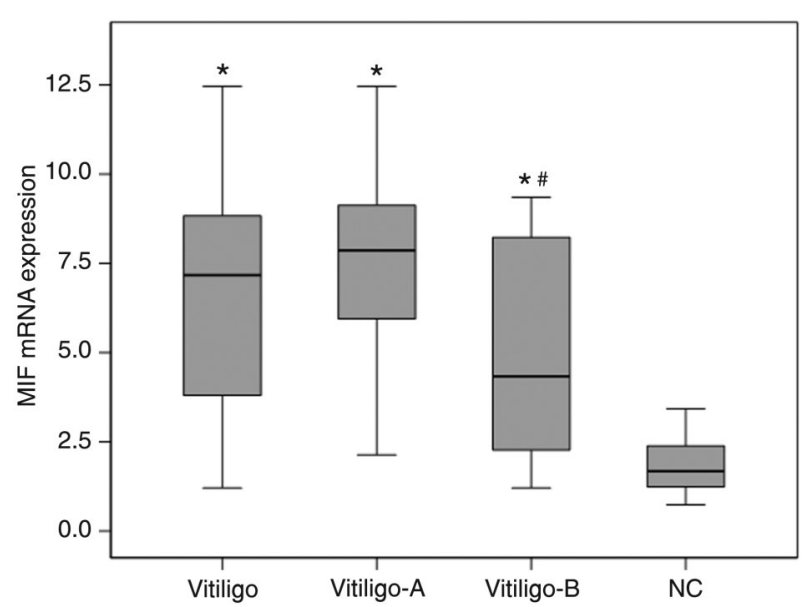

Figure 2. Migration inhibitory factor (MIF) mRNA levels in peripheral blood monnuclear cells from vitiligo vulgaris patients and normal controls. Vitiligo-A = Progressive vitiligo; Vitiligo-B = stable vitiligo. ${ }^{*} \mathrm{P}<0.01$ compared to normal controls $(\mathrm{NC})$; ${ }^{\#} \mathrm{P}<$ 0.05 compared to Vitiligo-A (Mann-Whitney U-test).

\section{Results}

\section{MIF expression is significantly high in vitiligo vulgaris patients}

As shown in Figures 2 and 3, MIF mRNA levels in PBMCs of patients and normal controls were 7.17 (3.59$8.87)$ and 1.67 (1.23-2.42), respectively, and the difference was statistically significant $(Z=-6.292, P<0.01)$. Serum MIF concentrations were significantly increased in patients with vitiligo vulgaris [35.81 (10.98-43.66) $\mathrm{ng} / \mathrm{mL}$ ] compared to control subjects [7.69 (6.01-9.03) ng/mL, Z $=-6.287, P<0.01]$. In another comparison, patients

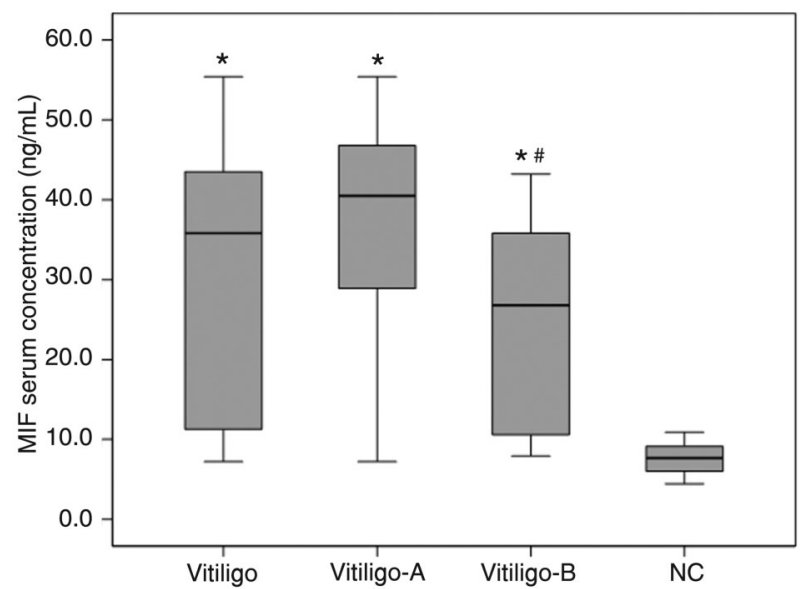

Figure 3. Serum migration inhibitory factor (MIF) concentrations of vitiligo vulgaris patients and normal controls. Vitiligo- $A=$ Progressive vitiligo; Vitiligo- $B=$ stable vitiligo. ${ }^{*} \mathrm{P}<0.01$ compared to normal controls (NC); ${ }^{\#} \mathrm{P}<0.05$ compared to Vitiligo-A (Mann-Whitney U-test). were subdivided according to disease activity and MIF mRNA levels in PBMCs and serum MIF concentrations of progressive and stable patients were all significantly higher than those of the control group. There was also a statistically significant difference between the two subgroup MIF mRNA levels in PBMCs, 7.86 (5.85-9.13) vs 4.33 (2.23-8.39), $\mathrm{Z}=-2.355, \mathrm{P}=0.019$; serum MIF concentrations, 40.47 (27.71-46.79) vs 26.80 (10.5536.07) $\mathrm{ng} / \mathrm{mL}, \mathrm{Z}=-2.489, \mathrm{P}=0.013$. MIF mRNA levels were significantly higher in lesional skin than in normal skin [2.43 (2.13-7.59) vs 1.18 (0.94-1.83), $Z=-2.959, \mathrm{P}$ $=0.003]$, and in the progressive stage than in the stable stage $[7.52(2.43-8.84)$ vs $2.13(1.98-2.64), Z=-2.548, P$ $=0.011]$.

\section{MIF levels correlated positively with VASI scores in vitiligo vulgaris patients}

Correlation analysis revealed that the VASI scores of vitiligo vulgaris patients correlated positively with changes of both MIF mRNA levels in PBMCs $(r=0.426, P=$ 0.004 ; Figure 4) and serum MIF concentrations ( $r=$ $0.488, P=0.001$; Figure 5).

\section{Discussion}

Vitiligo is a disorder of pigmentation characterized by the presence of depigmented skin macules, which has a negative impact on the psychological well-being and social interactions of the patients. The exact etiology and pathogenesis of vitiligo are not clear. Many theories have been put forward, among which the autoimmune theory is the most popular one, especially for vitiligo vulgaris. In addition, many studies have indicated a role for both cellular and humoral immunity in the pathogenesis

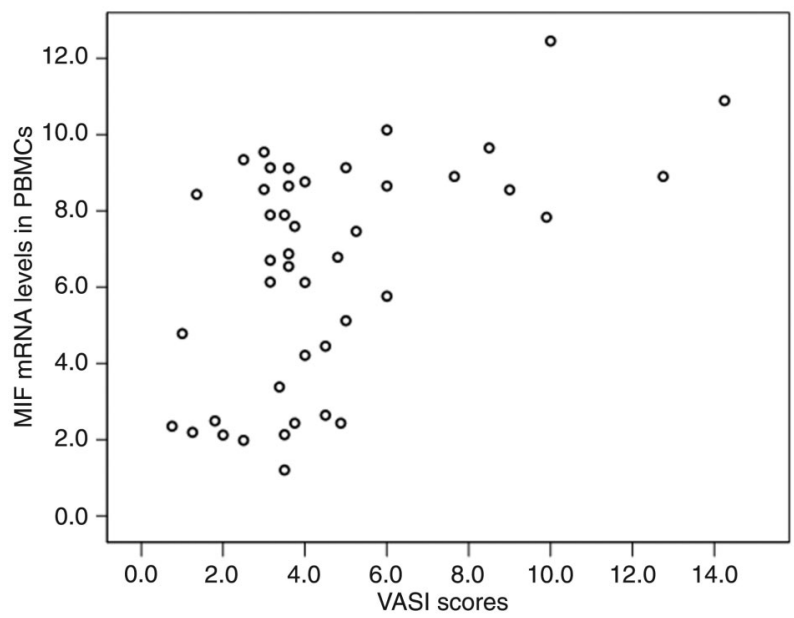

Figure 4. Correlation of migration inhibitory factor (MIF) mRNA levels in peripheral blood mononuclear cells (PBMCs) with vitiligo area severity index (VASI) scores of vitiligo vulgaris patients ( $r=$ $0.426, P=0.004$, Spearman's correlation test). 


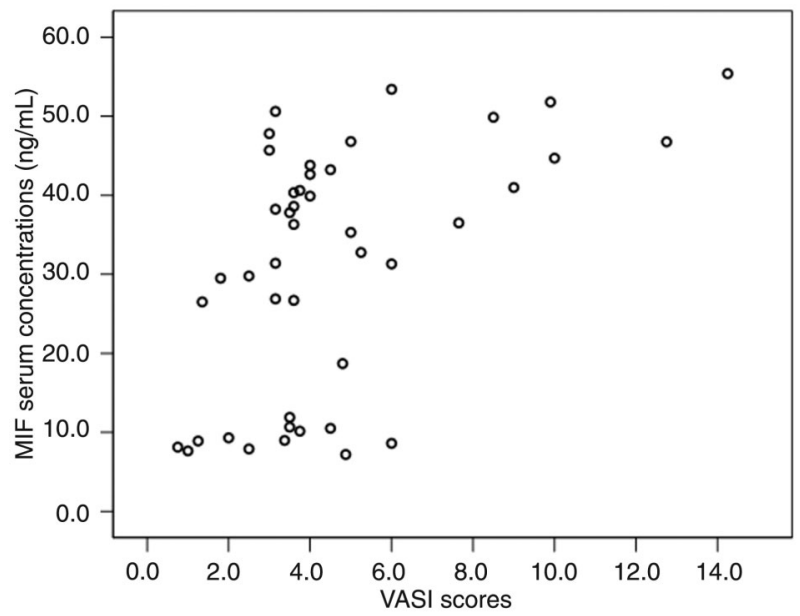

Figure 5. Correlation of serum migration inhibitory factor (MIF) concentrations with vitiligo area severity index (VASI) scores of vitiligo vulgaris patients $(r=0.488, P=0.001$, Spearman's correlation test).

of vitiligo (2). MIF has been shown to play a critical role in many immune/inflammatory skin diseases, including atopic dermatitis, bullous pemphigoid and psoriasis (7-10).

In the present study, we demonstrated that serum MIF concentrations were significantly elevated in most patients with vitiligo vulgaris, in agreement with the study from Serarslan et al. (12). Additionally, we measured the MIF mRNA levels in PBMCs and skin tissues for the first time and compared the relationship between MIF expressions and the disease severity and activity in vitiligo vulgaris. In parallel to the serological data, we identified over-expressions of MIF mRNA in both PBMCs and skin lesions, particularly in progressive patients, suggesting that MIF further participates in the occurrence and development of vitiligo vulgaris.

On the other hand, we observed that both MIF mRNA levels in PBMCs and serum MIF concentrations were positively associated with the VASI scores in vitiligo vulgaris, whereas Serarslan et al. (12) reported that there was no relationship between body surface area (BSA) and serum MIF levels in vitiligo. Some differences do exist in the measurement of skin lesional areas by different evaluators and, compared with the BSA scores, the VASI scores include both skin lesional areas and the degree of depigmentation, which may be the reasons for the difference between our results and those reported by

\section{References}

1. Kemp EH, Waterman EA, Weetman AP. Autoimmune aspects of vitiligo. Autoimmunity 2001; 34: 65-77, doi: 10.3109/08916930108994127.

2. Rezaei N, Gavalas NG, Weetman AP, Kemp EH. Autoimmunity as an aetiological factor in vitiligo. $J$ Eur
Serarslan et al.

It has been reported that macrophages are involved in clearing melanocytes in vitiligo. Macrophage infiltration has been demonstrated in vitiligo lesions and increased macrophage numbers are also observed in perilesional skin $(13,14)$. MIF can inhibit the random migration of macrophages, concentrate macrophages at inflammation loci and exert a variety of biological functions such as macrophage activation, enhancement of adherence, phagocytosis, and tumoricidal activity. In turn, macrophages are an important source of MIF. Thus, to some extent, it is possible that the MIF and macrophage loop may contribute to the pathogenesis of vitiligo.

In addition, TNF- $\alpha$ and IL- 6 are two inflammatory cytokines with an inhibitory effect on pigmentation. The expressions of TNF- $\alpha$ and IL- 6 were practically undetectable in the skin of normal subjects, whereas their mRNA levels were increased in the epidermis from vitiligo biopsies (15). Moreover, IL-6 has been shown to play an important role in melanocytic cytotoxicity, and increased serum IL-6 levels were also detected in vitiligo vulgaris patients (16).

MIF itself can function as an initial proinflammatory mediator, which is associated with the generation of cellmediated immune responses. Accordingly, MIF has been shown to induce up-regulation of many cytokines, including IL-1 $\beta$, IL-8, IFN- $\gamma$, TNF- $\alpha$, and IL-6 (17). Similarly, through decreased Toll-like receptor-4 expression and NF- $\kappa B$ activity, MIF-deficient macrophages show a reduced production of TNF, IL-1 and IL-6 (18). Therefore, as a pivotal immunomodulator in the cytokine network, MIF may induce the local inflammatory and immunological responses of depigmentation associated with vitiligo vulgaris in cooperation with other cytokines.

In conclusion, our results suggest that MIF may participate in the pathogenesis of vitiligo vulgaris and may act as an index of disease severity and activity.

\section{Acknowledgments}

Research supported by the Shandong Province Higher Educational Science and Technology Program of China (\#J10LF90 and \#J11LF66), the Shandong Province Natural Science Foundation of China (\#ZR2010HQ013) and the Scientific Research Foundation of Binzhou Medical University (\#BY2009KYQD03 and \#BY2010KYQD04).
Acad Dermatol Venereol 2007; 21: 865-876, doi: 10.1111/ j.1468-3083.2007.02228.x.

3. Spritz RA. The genetics of generalized vitiligo and associated autoimmune diseases. Pigment Cell Res 2007; 20: 271-278, doi: 10.1111/j.1600-0749.2007.00384.x. 
4. Shajil EM, Chatterjee S, Agrawal D, Bagchi T, Begum R. Vitiligo: pathomechanisms and genetic polymorphism of susceptible genes. Indian J Exp Biol 2006; 44: 526-539.

5. Huggins RH, Schwartz RA, Janniger CK. Vitiligo. Acta Dermatovenerol Alp Panonica Adriat 2005; 14: 137-142144-145.

6. Ingordo V, Gentile C, lannazzone SS, Cusano F, Naldi L. Vitiligo and autoimmunity: an epidemiological study in a representative sample of young Italian males. J Eur Acad Dermatol Venereol 2011; 25: 105-109, doi: 10.1111/j.14683083.2010.03696.x.

7. Shimizu T. Role of macrophage migration inhibitory factor (MIF) in the skin. J Dermatol Sci 2005; 37: 65-73, doi: 10.1016/j.jdermsci.2004.08.007.

8. Asano $\mathrm{Y}$, Makino $\mathrm{T}$, Norisugi $\mathrm{O}$, Watanabe $H$, Abe $\mathrm{R}$, Shimizu $\mathrm{H}$, et al. Macrophage migration inhibitory factor (MIF) in bullous pemphigoid. J Dermatol Sci 2008; 49: 9597, doi: 10.1016/j.jdermsci.2007.09.011.

9. Kim JY, Kwok SK, Hur KH, Kim HJ, Kim NS, Yoo SA, et al. Up-regulated macrophage migration inhibitory factor protects apoptosis of dermal fibroblasts in patients with systemic sclerosis. Clin Exp Immunol 2008; 152: 328-335, doi: 10.1111/j.1365-2249.2008.03637.x.

10. Selvi E, Tripodi SA, Catenaccio M, Lorenzini S, Chindamo $\mathrm{D}$, Manganelli $\mathrm{S}$, et al. Expression of macrophage migration inhibitory factor in diffuse systemic sclerosis. Ann Rheum Dis 2003; 62: 460-464, doi: 10.1136/ard.62.5.460.

11. Hamzavi I, Jain H, McLean D, Shapiro J, Zeng H, Lui H. Parametric modeling of narrowband UV-B phototherapy for vitiligo using a novel quantitative tool: the Vitiligo Area Scoring Index. Arch Dermatol 2004; 140: 677-683, doi: 10.1001/archderm.140.6.677.
12. Serarslan G, Yonden Z, Sogut S, Savas N, Celik E, Arpaci A. Macrophage migration inhibitory factor in patients with vitiligo and relationship between duration and clinical type of disease. Clin Exp Dermatol 2010; 35: 487-490, doi: 10.1111/j.1365-2230.2009.03617.x.

13. van den Wijngaard $R$, Wankowicz-Kalinska $A$, Le Poole $C$, Tigges B, Westerhof W, Das P. Local immune response in skin of generalized vitiligo patients. Destruction of melanocytes is associated with the prominent presence of CLA + T cells at the perilesional site. Lab Invest 2000; 80: 12991309, doi: 10.1038/labinvest.3780138.

14. Le Poole I, Van den Wijngaard RM, Westerhof W, Das PK. Presence of $\mathrm{T}$ cells and macrophages in inflammatory vitiligo skin parallels melanocyte disappearance. Am J Pathol 1996; 148: 1219-1228.

15. Moretti S, Fabbri P, Baroni G, Berti S, Bani D, Berti E, et al. Keratinocyte dysfunction in vitiligo epidermis: cytokine microenvironment and correlation to keratinocyte apoptosis. Histol Histopathol 2009; 24: 849-857.

16. Yu HS, Chang KL, Yu CL, Li HF, Wu MT, Wu CS, et al. Alterations in IL-6, IL-8, GM-CSF, TNF-alpha, and IFNgamma release by peripheral mononuclear cells in patients with active vitiligo. J Invest Dermatol 1997; 108: 527-529, doi: 10.1111/1523-1747.ep12289743.

17. Bucala R, Lolis E. Macrophage migration inhibitory factor: a critical component of autoimmune inflammatory diseases. Drug News Perspect 2005; 18: 417-426, doi: 10.1358/ dnp.2005.18.7.939345.

18. Roger T, David J, Glauser MP, Calandra T. MIF regulates innate immune responses through modulation of Toll-like receptor 4. Nature 2001; 414: 920-924, doi: 10.1038/ $414920 a$ 